\title{
The fructose-copper connection: Added sugars induce fatty liver and insulin resistance via copper deficiency
}

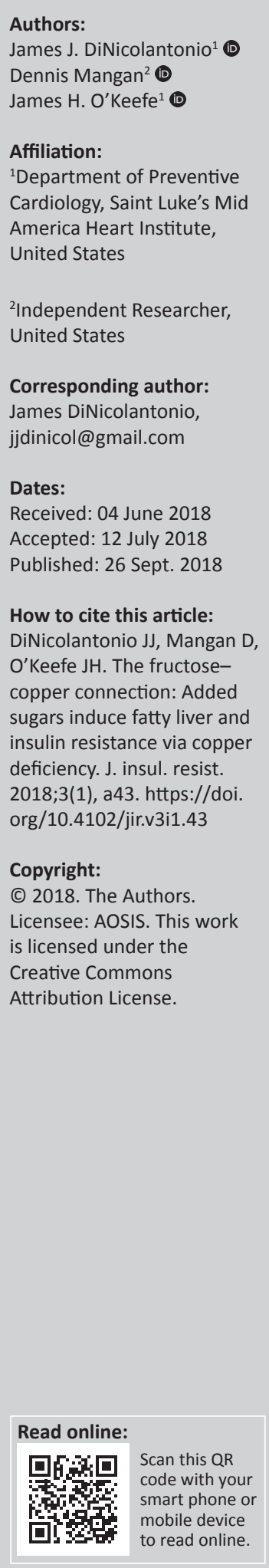

Background: Evidence suggests that the overconsumption of added sugars can induce fatty liver disease and insulin resistance.

Aim: To propose a hypothesis that added sugars induce copper deficiency which can lead to hepatic iron overload, fatty liver disease, insulin resistance and eventually non-alcoholic steatohepatitis.

Setting: On average, the intake of added sugars in humans is higher than levels that have been found to impair copper status in animals.

Methods: Narrative review.

Results: Fructose-induced copper deficiency may be a leading cause of fatty liver disease and insulin resistance.

Conclusion: The reduction in the intake of added sugars may improve copper status and reduce the risk of fatty liver disease and insulin resistance.

\section{Introduction}

The Western diet may be inadequate in copper with at least $25 \%$ of adults (but up to $80 \%$ or more) in the West consuming less than the estimated average requirement. ${ }^{1}$ Importantly, copper deficiency causes many of the risk factors and pathologies of ischemic heart disease (IHD), including increased serum triglycerides and cholesterol, hypertension, increased oxidation of low-density lipoprotein (LDL) cholesterol and vascular dysfunction. ${ }^{2}$ Consumption of sucrose (table sugar, which is 50\% fructose) is in turn associated with IHD with those in the highest levels of consumption of added sugars having a threefold higher risk of death because of cardiovascular disease. ${ }^{3}$ Sugar consumption is also associated with metabolic syndrome and non-alcoholic fatty liver disease ${ }^{4}$ (NAFLD); thus, feeding sugar to rats induces fatty liver. ${ }^{5}$ One of the mechanisms by which sugar leads to NAFLD may be by decreasing body copper stores and hence causing copper deficiency. This article will discuss the evidence regarding 'the fructose-copper connection' around NAFLD.

The prevalence of NAFLD has been increasing across the globe, and more so in developed nations with the incidence estimated to be about $20 \%$ of the United States adult population, but up to $30 \%$ of the general population in other Western countries. ${ }^{6,7}$ The causes of NAFLD are still the subject of debate, but it is associated with the metabolic syndrome and obesity.

\section{The fructose-copper connection}

The overconsumption of sugar may be one of the biggest drivers of NAFLD. ${ }^{4}$ Several factors may be involved in sugar-driven NAFLD, including bacterial translocation from the gut to the liver and advanced glycation end products. ${ }^{8}$ However, low hepatic copper levels are also implicated in NAFLD; thus sugar consumption can lead to low copper. ${ }^{9}$ This suggests that the overconsumption of sugar may lead to NAFLD and insulin resistance via hepatic copper deficiency.

In a rat model of NAFLD, dietary sucrose and copper deficiency increased inflammation, fibrosis and lipogenesis. ${ }^{9}$ Compared to a control diet in which rats were fed with sufficient copper and $10 \%$ sucrose, either a high-sucrose diet or a low copper diet increased the hepatic expression of genes regulating inflammation and fibrosis. The low copper diet led to low serum and hepatic 
copper, increased lipid peroxidation and histopathology similar to that found in NAFLD. The combined low copper and high sugar diet led to all of these changes as well as hepatic insulin resistance and liver damage, but neither low copper nor high sugar caused weight gain. Low copper and high sugar promoted gene expression and physiological processes typical of NAFLD as well as non-alcoholic steatohepatitis (NASH) even without weight gain.

The fructose component of dietary sucrose, in particular, can induce copper deficiency. In rats, a diet of only 3\% fructose from beverage intake - a rather modest fructose consumption that is lower than typical intake of Americans - impaired copper status and led to a significant induction of hepatic injury, iron overload and fat accumulation. ${ }^{10}$ Therefore, fructose-induced copper deficiency could be an important mechanism behind fructose-induced NAFLD. Ultra-high consumption of fructose is not necessarily required for fructose-induced fatty liver with levels lower than the average American consumption capable of inducing it in rats.

The feeding of fructose in rodents, in addition to inducing low copper status and hepatic copper deficiency, leads to a number of pathologies associated with low copper. These pathologies include iron overload, increased liver enzymes (aspartate aminotransferase or AST), increased hepatic triglycerides and increased hepatic fatty acid synthase. ${ }^{11}$ Fructose feeding also causes impaired expression of the copper transporter Ctr-1 in the duodenum and reducing copper absorption in the intestine which can explain the induction of copper deficiency by fructose. ${ }^{11}$ As ceruloplasmin (the main copper-containing enzyme in the blood) is involved in oxidation of iron from ferrous to the less toxic ferric form for loading on to transferrin, lack of copper, leading to decreased ceruloplasmin, may account for the iron overload seen in fatty liver. ${ }^{12}$ Indeed, hereditary copper deficiency leads to liver iron overload. ${ }^{13}$

Perturbations in hepatic iron are seen frequently in NAFLD. Patients with NAFLD have lower hepatic copper, and those with lower levels of both hepatic copper and serum copper, have higher ferritin levels $(606 \mathrm{mg} / \mathrm{L}$ vs. $224 \mathrm{mg} / \mathrm{L}$, a 2.7-fold difference), higher hepatic iron concentrations $(1184 \mu \mathrm{g} / \mathrm{g}$ vs. $320 \mu \mathrm{g} / \mathrm{g}$, 3.7-fold higher), lower concentrations of ceruloplasmin and decreased liver gene expression for ferroportin, the cellular iron exporter. ${ }^{14}$ Taken together, these results suggest that (1) copper status is linked to iron status in NAFLD; (2) a significant number of NAFLD patients are deficient in copper; and (3) low copper increases hepatic iron load by decreasing ferroportin levels and the ferroxidase function via lower ceruloplasmin which blocks liver iron export. Thus, copper deficiency may lead to hepatic iron overload causing NAFLD, NASH and hepatic insulin resistance.

High-fructose-induced copper deficiency in rats can also induce oxidative damage in the liver that does not appear to be wholly iron dependent, indicating some other cause of free-radical damage possibly via lower ceruloplasmin concentrations. ${ }^{15}$

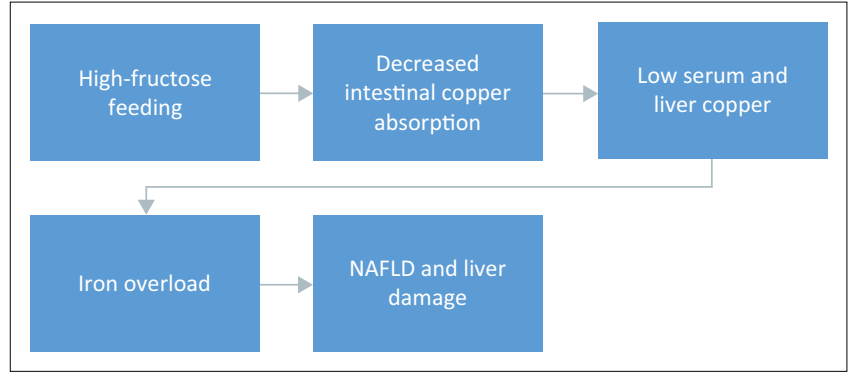

NAFLD, non-alcoholic fatty liver disease.

FIGURE 1: How high dietary fructose leads to copper deficiency and liver damage.

Another strategy for lowering iron levels, besides increasing copper status, is phlebotomy which can significantly treat NAFLD by improving insulin resistance. ${ }^{16}$ In Ctr- 1 (copper transporter) knockout mice, defects in copper absorption lead to hepatic iron overload, along with cardiac hypertrophy and growth defects. ${ }^{17}$ A single administration of copper can rescue many of these defects. Patients with iron overload of even mild to moderate levels almost universally have some type of insulin resistance syndrome. ${ }^{18}$ Low dietary copper, whether from deficient diets or high fructose intake, may play a key role in the high prevalence of insulin resistance and NAFLD.

Although copper deficiency can lead to glucose intolerance, this phenomenon appears to occur only with simultaneous feeding of glucose or fructose, but not with starch. ${ }^{19}$ The average intake of added sugars among Americans is anywhere from $15 \%$ to $30 \%$ of calories (based on 2500 calories consumed per day). ${ }^{20}$ As noted above, even a modest (3\%) intake of fructose in rats can lead to copper deficiency. Thus, the amount of sugar consumed by the average American seems to be high enough to induce copper deficiency.

Feeding of isolated cholesterol in animals can also induce copper deficiency, and in turn, deficiency of copper can induce hypercholesterolemia as well as glucose intolerance and hypertriglyceridemia. ${ }^{21,22}$ Copper deficiency is one of the only, perhaps the only, nutritional deficiency that increases cholesterol, blood pressure and uric acid, and promotes glucose intolerance and oxidative damage, all of which are associated with atherosclerosis. ${ }^{21}$ Diets which induce copper deficiency, resemble the Western diet, which is high in sugars and fat, and low in copper.

Copper deficiency may play an important, if not the major, part in the promotion of NAFLD, insulin resistance and atherosclerosis. Known risk factors for heart disease which can be induced by copper deficiency, include elevated triglycerides, hypertension and glucose intolerance, all of which are common in the American population. High consumption of fructose leading to copper deficiency or diets inadequate in copper, could be the 'missing link' in NAFLD, insulin resistance and IHD, one that would be easily remedied by better food choices (such as shellfish, organ meats, oysters, nuts, seeds and beans) or even merely a copper supplement. ${ }^{23}$ 


\section{Conclusion}

The Western diet is loaded with fructose via the consumption of added sugars. Recent estimates put sugar consumption at $15 \%-30 \%$ of calories consumed with $13 \%$ of the population consuming at least $25 \%$ of their calories from added ${ }^{20}$ with a higher percentage consumption in adult females (14.5\%) and young adult males (14.1\%). Those figures approximate to an average consumption in the United States between $77 \mathrm{lbs}$ and 152 lbs. of added sugars per person per year. ${ }^{20}$ The Western diet is also low in copper with at least $25 \%$ of people failing to ingest the estimated average requirement for copper.

The Western world is currently in the throes of an epidemic of obesity and diabetes, and along with it, fatty liver. The rest of the world is catching up in incidence of these diseases. Fructose, via the induction of copper deficiency, could be driving these conditions, along with the well-known and long-standing epidemic of heart disease. The evidence presented here shows that high sugar consumption with resultant copper deficiency could well be a very important factor in these diseases of civilisation. More research is needed to (1) to delineate the degree to which the average person is copper sufficient or insufficient; (2) study the degree to which groups of people with or without these diseases differ in levels of body copper stores and dietary copper intake; (3) study trials of copper supplementation and/or prospective studies of copper status and ensuing pathologies; and (4) explain mechanisms of fructose-induced copper deficiency and iron overload.

\section{Acknowledgements Competing interests}

The authors declare that they have no financial or personal relationships which may have inappropriately influenced them in writing this article.

\section{Authors' contributions}

J.J.D. performed the literature search. All authors contributed to edits and to the writing of the article.

\section{References}

1. Klevay LM. Is the Western diet adequate in copper? J Trace Elem Med Biol. 2011;25:204-212. https://doi.org/10.1016/j.jtemb.2011.08.146
2. DiNicolantonio J, Mangan D, O'Keefe JH. Copper deficiency may be a leading cause of Ischemic heart disease. Open Heart. 2018; In press.

3. DiNicolantonio JJ, Lucan SC, O'Keefe JH. The evidence for saturated fat and for sugar related to coronary heart disease. Prog Cardiovasc Dis. 2015, 58(5):464-72.

4. DiNicolantonio JJ, Subramonian AM, O'Keefe JH. Added fructose as a principal driver of non-alcoholic fatty liver disease: A public health crisis. Open Heart. 2017;4:e000631. https://doi.org/10.1136/openhrt-2017-000631

5. Sanchez-Lozada LG, Mu W, Roncal C, et al. Comparison of free fructose and glucose to sucrose in the ability to cause fatty liver. Eur J Nutr. 2010;49:1-9. https://doi.org/10.1007/s00394-009-0042-x

6. Lazo M, Hernaez R, Eberhardt MS, et al. Prevalence of nonalcoholic fatty liver disease in the United States: The Third National Health and Nutrition Examination Survey, 1988-1994. Am J Epidemiol. 2013;178:38-45. https://doi.org/10.1093/ aje/kws448

7. Lazo M, Hernaez R, Bonekamp S, et al. Non-alcoholic fatty liver disease and mortality among US adults: Prospective cohort study. BMJ. 2011;343:d6891. https://doi.org/10.1136/bmj.d6891

8. Yilmaz Y. Review article: Fructose in non-alcoholic fatty liver disease. Aliment Pharmacol Ther. 2012;35:1135-1144. https://doi.org/10.1111/j.1365-2036.2012. 05080.x

9. Tallino S, Duffy $M$, Ralle $M$, et al. Nutrigenomics analysis reveals that copper deficiency and dietary sucrose up-regulate inflammation, fibrosis and lipogenic pathways in a mature rat model of nonalcoholic fatty liver disease. J Nutr Biochem. 2015;26:996-1006. https://doi.org/10.1016/j.jnutbio.2015.04.009

10. Song M, Schuschke DA, Zhou Z, et al. Modest fructose beverage intake causes liver injury and fat accumulation in marginal copper deficient rats. Obesity (Silver Spring, MD). 2013;21:1669-1675. https://doi.org/10.1002/oby.20380

11. Song M, Schuschke DA, Zhou Z, et al. High fructose feeding induces copper deficiency in Sprague-Dawley rats: A novel mechanism for obesity related fatty liver. J Hepatol. 2012;56:433-440. https://doi.org/10.1016/j.jhep.2011.05.030

12. Young SP, Fahmy M, Golding S. Ceruloplasmin, transferrin and apotransferrin facilitate iron release from human liver cells. FEBS Lett. 1997;411:93-96. https:// doi.org/10.1016/S0014-5793(97)00478-X

13. Nittis T, Gitlin JD. The copper-iron connection: Hereditary aceruloplasminemia Semin Hematol. 2002;39:282-289. https://doi.org/10.1053/shem.2002.35633

14. Aigner $E$, Theurl I, Haufe $H$, et al. Copper availability contributes to iron perturbations in human nonalcoholic fatty liver disease. Gastroenterology. 2008;135:680-688. https://doi.org/10.1053/j.gastro.2008.04.007

15. Fields $M$, Lewis $C G$, Lure $M$, et al. The influence of gender on developing copper deficiency and on free radical generation of rats fed a fructose diet. Metabolism. 1992;41:989-994. https://doi.org/10.1016/0026-0495(92)90125-T

16. Valenti L, Fracanzani AL, Dongiovanni $\mathrm{P}$, et al. Iron depletion by phlebotomy improves insulin resistance in patients with nonalcoholic fatty liver disease and hyperferritinemia: Evidence from a case-control study. Am J Gastroenterol. 2007;102:1251-1258. https://doi.org/10.1111/j.1572-0241.2007.01192.x

17. Nose $Y, K i m$ BE, Thiele DJ. Ctr1 drives intestinal copper absorption and is essential for growth, iron metabolism, and neonatal cardiac function. Cell Metabol. 2006;4:235-244. https://doi.org/10.1016/j.cmet.2006.08.009

18. Mendler $\mathrm{MH}$, Turlin $\mathrm{B}$, Moirand $\mathrm{R}$, et al. Insulin resistance-associated hepatic iron overload. Gastroenterology. 1999;117:1155-1163. https://doi.org/10.1016/S00165085(99)70401-4

19. Fields M, Ferretti RJ, Smith JC, Jr., et al. Impairment of glucose tolerance in copperdeficient rats: Dependency on the type of dietary carbohydrate. J Nutr. 1984;114:393-397. https://doi.org/10.1093/jn/114.2.393

20. DiNicolantonio JJ, Lucan SC. The wrong white crystals: Not salt but sugar as aetiological in hypertension and cardiometabolic disease. Open Heart. 2014;1. https://doi.org/10.1136/openhrt-2014-000167

21. Klevay LM. Metabolic interactions among dietary cholesterol, copper, and fructose. Am J Physiol Endocrinol Metab. 2010;298:E138-E1389. https://doi. org/10.1152/ajpendo.00591.2009

22. Klevay LM. Ischemic heart disease. A major obstacle to becoming old. Clin Geriatr Med. 1987;3:361-372. https://doi.org/10.1016/S0749-0690(18)30817-6

23. Aliabadi H. A deleterious interaction between copper deficiency and sugar ingestion may be the missing link in heart disease. Med Hypotheses. 2008;70:1163-1166. https://doi.org/10.1016/j.mehy.2007.09.019 